\title{
BMJ open Coronary Artery Bypass Grafting Surgery Off- or On-pump Revascularisation Study (CORONARY): kidney substudy analytic protocol of an international randomised controlled trial
}

Amit X Garg, ${ }^{1,2,3}$ P J Devereaux, ${ }^{3,4}$ Salim Yusuf, ${ }^{3,4}$ Meaghan S Cuerden, ${ }^{1,5}$ Chirag R Parikh, ${ }^{6}$ Steven G Coca, ${ }^{6}$ Michael Walsh, ${ }^{3,4}$ Richard J Cook, ${ }^{5}$ Richard P Whitlock, ${ }^{3,4,7}$ Nicolas Noiseux, ${ }^{8}$ Richard J Novick, ${ }^{9}$ Yongning Ou, ${ }^{4}$ Andre Lamy, ${ }^{3,4,7}$ on behalf of the CORONARY Investigators

To cite: Garg AX, Devereaux PJ, Yusuf S, et al. Coronary Artery Bypass Grafting Surgery Off- or On-pump Revascularisation Study (CORONARY): kidney substudy analytic protocol of an international randomised controlled trial. BMJ Open 2012;2:e001080.

doi:10.1136/

bmjopen-2012-001080

- Prepublication history and additional appendix for this paper are available online. To view these files please visit the journal online (http://dx. doi.org/10.1136/

bmjopen-2012-001080).

Received 28 February 2012 Accepted 6 March 2012

This final article is available for use under the terms of the Creative Commons Attribution Non-Commercial 2.0 Licence; see http://bmjopen.bmj.com

For numbered affiliations see end of article.

Correspondence to Dr Amit X Garg; amit.garg@|hsc.on.ca

\section{ABSTRACT}

Introduction: CORONARY is a large international randomised controlled trial comparing coronary artery bypass graft (CABG) surgery done with and without a bypass pump. Compared with on-pump, off-pump surgery may prevent acute kidney injury (AKI) in the short term and may better preserve kidney function 1 year following surgery. Secondary analyses may also clarify whether effects are similar in patients with and without pre-operative chronic kidney disease and whether AKI avoidance mediates preserved 1-year kidney function.

Methods and analysis: With respect to the study schedule, the last of 4752 patients from 79 sites in 19 countries were randomised in November 2011 to cardiac surgery performed with an on-pump or off-pump procedure. The authors will use regression models to compare the groups in the outcome of peri-operative AKI (per cent change in serum creatinine, $\geq 50 \%$ increase in serum creatinine) and 1-year kidney function (per cent change in estimated glomerular filtration rate (eGFR), $\geq 20 \%$ eGFR loss 1 year after surgery). The authors will use interaction terms in regression models to determine if there is a differential impact of the intervention in those with and without pre-existing chronic kidney disease. The authors will use regression-based tests to determine the proportion of the total effect of surgery type (off-pump vs on-pump CABG) on 1-year eGFR that is mediated by peri-operative AKI.

Ethics and dissemination: In the year 2009, the authors were competitively awarded a grant from the Canadian Institutes of Health Research to answer these kidney questions in CORONARY. Ethics approval was obtained for additional renal data collection in centres that agreed to study participation ( $>90 \%$ of participating centres). This collection began for patients enrolled after 1 January 2010. Remaining 1-year renal outcome data will be collected throughout 2012. Results will be reported in 2013.

Clinical trial registration number: NCT 00463294.

\section{ARTICLE SUMMARY}

Article focus

- CORONARY is a large international randomised controlled trial comparing coronary artery bypass graft (CABG) surgery done with and without a bypass pump.

- Compared with on-pump, off-pump surgery may prevent AKI in the short term and may better preserve kidney function 1 year after surgery.

- Secondary analyses may also clarify whether effects are similar in patients with and without pre-operative chronic kidney disease and whether AKI avoidance mediates preserved 1-year kidney function.

Key messages

- Presented is this pre-specified CORONARY kidney substudy analytic protocol.

- Data collection and analysis will be completed in 2013.

- Understanding the degree to which avoiding AKI preserves longer term kidney function has broader implications for the acceptability of side effects and costs of interventions which prevent AKI.

Strengths and limitations of this study

- This will be largest AKI prevention trial conducted to date.

- It will be one of the first trials to consider the impact of a peri-operative intervention on longer term kidney function.

- International recruitment across 19 countries will provide generalisable estimates of the treatment effect.

\section{BACKGROUND}

Coronary artery bypass grafting (CABG) surgery is an effective way of improving 
symptoms and prolonging life in patients with severe coronary artery disease. ${ }^{1}$ Globally, 1.25 million cardiac surgeries are performed annually. ${ }^{2}$ A frequent and serious complication of CABG surgery is acute kidney injury (AKI), which is a sudden deterioration in kidney function. Peri-operative AKI is independently associated with increased short- and long-term mortality, a greater length of hospital stay and higher economic costs. ${ }^{3}$

CABG surgery is usually performed with the use of cardiopulmonary bypass ('on-pump'). This requires cannulation of the heart and aorta, cross clamping of the ascending aorta and cardioplegic arrest. In this setting, AKI is multifactorial: the bypass circuit changes blood concentration and pulsatility and introduces a blood/ circuit interface, which stimulates a generalised inflammatory response including increased catecholamine and free haemoglobin levels. ${ }^{4}$ Furthermore, aorta cannulation and cross clamping may lead to platelet or atheromatous embolic phenomena.

CABG surgery performed on a beating heart without the use of cardiopulmonary bypass ('off-pump') was developed to avoid post-operative complications from the pump. A systematic review published in 2010 evaluated AKI across 22 randomised trials comparing off-pump with on-pump surgery (total 4819 patients). ${ }^{5}$ Off-pump CABG resulted in a $40 \%$ RR reduction in AKI (variably defined in the primary trials). The results did not demonstrate statistical heterogeneity. However, as concluded by the review authors, 'different definitions of AKI used in the individual trials and methodological concerns preclude definitive conclusions about the treatment effect'.

A limitation of most AKI prevention trials is a failure to consider any sustained effect of the intervention on longer term kidney function. It is possible that off-pump compared with on-pump surgery better preserves 1-year kidney function, but to our knowledge, this has not been previously evaluated. This effect may be mediated through the AKI itself (ie, avoiding AKI preserves kidney function at 1 year ${ }^{6}$ ) or may be mediated through other pathways (ie, off-pump bypass improves cardiac function which preserves 1-year kidney function). Understanding the degree to which avoiding AKI preserves longer term kidney function has broader implications for the acceptability of side effects and costs of interventions which prevent AKI. Finally, patients with pre-operative chronic kidney disease (CKD) are at high risk of AKI and are a subgroup where preservation of renal function is of key interest. ${ }^{8}$

\section{CABG Off- or On-Pump Revascularisation Study}

We are conducting the CABG Off- or On-Pump Revascularisation Study (acronym CORONARY). The methods of this large, international randomised controlled trial are described elsewhere. ${ }^{9}$ In brief, after obtaining written informed consent, adult patients undergoing isolated CABG surgery (with a median sternotomy) are allocated to have the procedure done with or without a bypass pump. Allocation is done by a voice-activated telephone randomisation service. This trial is funded by the Canadian Institutes of Health Research. With respect to the study schedule, the last of 4752 patients from 79 sites in 19 countries were randomised in November 2011. The primary 30-day composite outcome is total mortality, stroke, non-fatal myocardial infarction or receipt of dialysis for severe AKI. The 30-day results will be analysed and reported in March 2012. One-year outcome data will continue to be collected throughout 2012 and will be reported in 2013.

In addition to the primary outcome, CORONARY is uniquely positioned to answer important kidney-specific questions. In the year 2009, we were competitively awarded another grant from the Canadian Institutes of Health Research to collect additional renal information within CORONARY to address these questions. Ethics approval was obtained for additional renal data collection in centres that agreed to study participation $(>90 \%$ of participating centres). This collection began for patients enrolled after 1 January 2010. Remaining 1-year renal outcome data will be collected throughout 2012 . Results will be reported in 2013.

The kidney questions detailed in the grant are presented below and are followed by pre-specified analytic plans.

\section{Primary questions}

1. In patients undergoing CABG surgery, does use of an off-pump compared with an on-pump procedure (i) reduce the risk of AKI during the hospital stay and (ii) result in better kidney function 1 year after surgery?

\section{Secondary questions}

2. Does the presence of pre-operative CKD modify the impact of surgery type (off-pump, on-pump) on kidney outcomes?

3. If an off-pump compared with an on-pump procedure results in better kidney function 1 year after surgery is the effect mediated by avoiding peri-operative AKI?

\section{CORONARY data collection}

The pre-operative serum creatinine value (within 7 days prior to randomisation) has been recorded since the start of the trial, along with the peak value during the hospital stay. We define pre-operative CKD as an estimated glomerular filtration rate (eGFR) $<60 \mathrm{ml} / \mathrm{min} / 1.73 \mathrm{~m}^{2}$, using the Chronic Kidney Disease Epidemiology Collaboration (CKD-EPI) equation (which requires knowledge of whether a patient is of black race; $<0.1 \%$ of CORONARY patients). ${ }^{10}$ In centres that agreed to additional renal data collection, we started recording all serum creatinine measurements during the hospital stay for patients enrolled after 1 January 2010 (not just the peak value) and a serum creatinine 1 year after surgery.

\section{ANALYTIC PLAN}

To refine the analytic plan in December 2011, we reviewed all CORONARY data without knowledge of 
patient allocation. Table 1 presents AKI according to modern staging systems. ${ }^{11} 12$ Table 2 presents changes in kidney function 1 year after surgery. The analytic plan was finalised without any knowledge of CORONARY outcomes by allocation group.

\section{Patient selection}

For CORONARY kidney substudy analyses, all randomised patients will be included except as follows: (1) patients receiving chronic haemodialysis prior to randomisation (as these patients cannot develop AKI - to date $1.3 \%$ of randomised patients), (2) patients with a baseline eGFR $<15 \mathrm{ml} / \mathrm{min} / 1.73 \mathrm{~m}^{2}$ prior to randomisation (ie, patients with end-stage renal disease (ESRD $)^{13}$; to date an additional $0.2 \%$ of patients), (3) patients missing a pre-randomisation serum creatinine value as we cannot reliably define AKI without knowledge of the baseline value $(<1 \%$ of patients) and (4) patients who never undergo CABG surgery as they will not have the opportunity to have any post-operative serum creatinine measurements ( $<1 \%$ of randomised patients).

\section{Intervention group assignment}

Patients are randomised to on-pump or off-pump CABG, with randomisation stratified by centre. The intentionto-treat principle will guide all analyses, irrespective of whether there is a deviation from the randomly allocated therapy. Currently, the cross-over rate is $4 \%$ from onpump to off-pump and $10 \%$ from off-pump to on-pump surgery.

\section{AKI during hospital stay}

Peri-operative per cent change in serum creatinine

We will use a linear regression model to compare the groups in the outcome of per cent change in serum creatinine ((peak post-operative serum creatinine- pre-randomisation serum creatinine)/pre-randomisation serum creatinine), stratified by centre and adjusting for the following covariates: age (per year), sex, left ventricular function categories $(\geq 50 \%, 35 \%-49 \%$, $20 \%-34 \%,<20 \%$ ), diabetes, pre-randomisation ACE inhibitor or angiotensin receptor blocker use, prerandomisation statin use, pre-randomisation diuretic use, urgent versus elective surgery and pre-randomisation eGFR category $\left(>60 \mathrm{ml} / \mathrm{min} / 1.73 \mathrm{~m}^{2}, \leq 60 \mathrm{ml} / \mathrm{min} /\right.$ $\left.1.73 \mathrm{~m}^{2}\right)$. We will include a missing data indicator value for each covariate (at present, there is $<1 \%$ missing for each variable). ${ }^{14}$ In patients who underwent surgery but have a missing post-operative peak serum creatinine value ( $<4 \%$ patients), we will carry the pre-randomisation serum creatinine forward as the post-operative value (which should provide a more conservative estimate of the intervention effect than the alternative of removing such patients). We will test model assumptions (detailed in online appendix) and interpret a $p$ value $\leq 0.05$ as statistically significant. We will report the result as the average difference in per cent change in serum creatinine between the surgical groups with $95 \%$ CI. Visually the unadjusted results will be graphed as box-plots. A sample of $\sim 4700$ patients will have over $80 \%$ power to detect a $5 \%$ or greater difference in the mean per cent change in serum creatinine between the two groups $(\alpha 0.05$, independent samples $\mathrm{t}$ test; adequate power to detect a small effect in relation to expected SD of 60).

\section{Categorised AKI}

We will use a logistic regression model to compare the groups in the outcome of $\geq 50 \%$ increase in serum creatinine, stratified by centre and adjusting for previously defined covariates ${ }^{111516}$ We will test model assumptions (detailed in online appendix) and will estimate the adjusted RR of AKI with $95 \%$ CI (bootstrap method

Table 1 Per cent of CORONARY patients to date who met a definition of acute kidney injury according to modern staging systems

\begin{tabular}{|c|c|c|c|}
\hline & $\begin{array}{l}\text { All patients } \\
(n=3089)\end{array}$ & $\begin{array}{l}\text { Patients with } \\
\text { a pre-operative } \\
\text { eGFR }>60 \mathrm{ml} / \mathrm{min} / \\
1.73 \mathrm{~m}^{2}(\mathrm{n}=2372)\end{array}$ & $\begin{array}{l}\text { Patients with } \\
\text { a pre-operative } \\
\text { eGFR } \leq 60 \mathrm{ml} / \mathrm{min} / \\
1.73 \mathrm{~m}^{2}(\mathrm{n}=717)\end{array}$ \\
\hline $\begin{array}{l}\text { Evidence of an absolute increase in } \mathrm{SCr} \text { value } \geq 27 \mu \mathrm{mol} / \mathrm{L} \\
\text { or an increase of } \geq 150 \% \text { from the baseline } \mathrm{SCr} \text { value } \\
\text { (AKIN stage } 1 \text { or more) }\end{array}$ & $28.9 \%$ & $25.3 \%$ & $41.1 \%$ \\
\hline $\begin{array}{l}\text { Evidence of an increase in SCr value } \geq 150 \% \text { ( } \geq 1.5 \text {-fold) } \\
\text { from baseline (RIFLE risk category) }\end{array}$ & $18.7 \%$ & $17.0 \%$ & $24.4 \%$ \\
\hline $\begin{array}{l}\text { Evidence of an increase in SCr value } \geq 200 \% \text { ( } \geq \text { twofold) } \\
\text { from baseline (RIFLE injury category, AKIN stage } 2 \text { ) }\end{array}$ & $6.9 \%$ & $5.6 \%$ & $11.4 \%$ \\
\hline $\begin{array}{l}\text { Evidence of an increase in SCr value } \geq 300 \% \text { ( } \geq \text { threefold) } \\
\text { from baseline or a baseline SCr } \geq 354 \mu \mathrm{mol} / \mathrm{l} \text { with an } \\
\text { increase } \geq 44 \mu \mathrm{mol} / \mathrm{I} \text { from baseline. Any patient who } \\
\text { received acute dialysis is categorised in this category } \\
\text { (RIFLE failure category, AKIN stage } 3 \text { ) }\end{array}$ & $2.2 \%$ & $1.6 \%$ & $4.2 \%$ \\
\hline Receipt of acute dialysis & $1.2 \%$ & $0.6 \%$ & $3.1 \%$ \\
\hline
\end{tabular}


Table 2 One-year change in eGFR compared with the pre-operative value, expressed as categories

\begin{tabular}{|c|c|c|c|}
\hline & $\begin{array}{l}\text { All patients } \\
(n=1241)\end{array}$ & $\begin{array}{l}\text { Patients with a pre-operative } \\
\text { eGFR }>60 \mathrm{ml} / \mathrm{min} / \\
1.73 \mathrm{~m}^{2}(\mathrm{n}=910)\end{array}$ & $\begin{array}{l}\text { Patients with a pre-operative } \\
\text { eGFR } \leq 60 \mathrm{ml} / \mathrm{min} / \\
1.73 \mathrm{~m}^{2}(\mathrm{n}=331)\end{array}$ \\
\hline$\geq 15 \%$ reduction in eGFR & $23 \%$ & $24 \%$ & $21 \%$ \\
\hline$\geq 20 \%$ reduction in eGFR & $18 \%$ & $19 \%$ & $15 \%$ \\
\hline$\geq 25 \%$ reduction in eGFR & $12 \%$ & $12 \%$ & $11 \%$ \\
\hline$\geq 50 \%$ reduction in eGFR & $3 \%$ & $2 \%$ & $5 \%$ \\
\hline$\geq 5 \mathrm{ml} / \mathrm{min} / 1.73 \mathrm{~m}^{2}$ reduction in eGFR & $38 \%$ & $43 \%$ & $24 \%$ \\
\hline$\geq 10 \mathrm{ml} / \mathrm{min} / 1.73 \mathrm{~m}^{2}$ reduction in eGFR & $25 \%$ & $29 \%$ & $15 \%$ \\
\hline
\end{tabular}

detailed in online appendix). We will have over $90 \%$ power to detect a $20 \%$ or more RR reduction should it exist (two-tailed $\alpha 0.05, \chi^{2}$ test). Of note, many factors including haemodilution add 'noise' to post-operative serum creatinine measurements. When the value is markedly elevated, we are confident that the reason is due to AKI. It is conceivable that an intervention effect will be observed for categorised AKI but not per cent change in creatinine. If the $p$ value is greater for per cent change than categorised AKI, we will interpret a $p$ value $\leq 0.025$ for categorised AKI as statistically significant. ${ }^{17}$ Because categorised AKI is easy to interpret, if it meets criteria for significance, we will primarily focus on this outcome.

\section{Supporting analyses}

We will perform six analyses defining AKI in other ways. We will interpret a $\mathrm{p}$ value $\leq 0.05$ as significant provided there is concordance with the primary results.

- Absolute change in serum creatinine from the pre-randomisation value.

- $\geq 100 \%$ increase in serum creatinine (Risk, Injury, Failure, Loss and End-stage Renal Disease (RIFLE) injury category, AKIN (Acute Kidney Injury Network) stage 2). ${ }^{11} 12$

- $\geq 27 \mu \mathrm{mol} / \mathrm{l}$ or $\geq 50 \%$ increase in serum creatinine (AKIN stage 1 or more). ${ }^{12}$

- Composite of AKI or death during the hospital stay (death rate $\sim 2 \%$ at 30 days; $90 \%$ of patients who die have post-operative serum creatinine recorded; death rate within 2 days of surgery is $<0.5 \%$ ).

- Evidence of a rise in serum creatinine in first $48 \mathrm{~h}$ after surgery (for patients enrolled after 1 January 2010). ${ }^{18}$

- AKI that also considers later post-operative rises in serum creatinine in relation to earlier post-operative measurements (supplements the primary analysis which only considers peak post-operative measurement during hospital stay in relation to pre-randomisation value). ${ }^{18}$

\section{Kidney function 1 year after surgery}

One-year per cent change in eGFR

Similar to the previously described AKI analysis, we will use a linear regression model to compare the groups in the outcome of per cent change in eGFR 1 year after surgery ((post-operative eGFR measured 1 year after surgery-pre-randomisation eGFR)/pre-randomisation eGFR), stratified by centre and adjusting for covariates described for AKI. In patients with missing 1-year values ( $\sim 5 \%$ for reasons of death, target $<10 \%$ for reasons of missing data), we will substitute the pre-randomisation eGFR value. We will impute an eGFR value of $5 \mathrm{ml} / \mathrm{min} /$ $1.73 \mathrm{~m}^{2}$ for any patient who developed ESRD anytime in follow-up $(<0.1 \%)$ or who died after receiving acute dialysis ( $\sim 0.6 \%$ patients $)$. With at least 1800 patients, we will have over $80 \%$ power to detect a difference of $4 \%$ or more between the two surgical groups $(\alpha 0.05$, independent samples t test, adequate power to detect a small to moderate effect in relation to expected SD of 25).

\section{Categorised change in kidney function}

Similar to the previously described AKI analysis, we will use a logistic regression model to compare the groups on the outcome of $\geq 20 \%$ eGFR decrease 1 year after surgery (ie, loss of over a fifth of kidney function). Anyone who develops ESRD in follow-up, or receives acute dialysis and dies before a serum creatinine measurement can be obtained, will be counted as having met this definition. We will have between $75 \%$ and $89 \%$ power to detect a $25 \%-30 \% \mathrm{RR}$ reduction should it exist (two-tailed $\propto 0.05, \chi^{2}$ test). As with AKI, it is conceivable that an intervention effect will be observed in the categorised but not continuous outcome. In this scenario, we will interpret statistical significance as described for AKI.

\section{Supporting analyses}

We will perform two analyses examining 1-year kidney function in other ways. We will interpret a $p$ value $\leq 0.05$ as significant provided there is concordance with the primary results.

- Absolute change in serum creatinine from the pre-randomisation value.

- Rank-based assessment accounting for potential competing event of death (detailed in online appendix).

\section{Subgroup analyses: presence of pre-operative CKD}

We will use interaction terms in the previously described linear and logistic regression models to determine if 


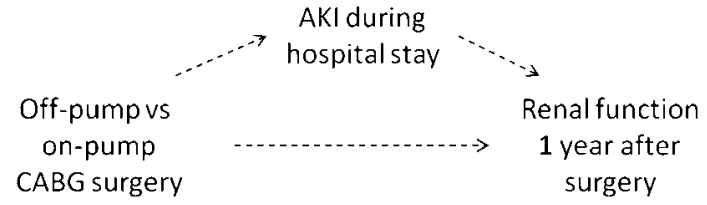

Figure 1 Directed acyclic graph of the causal pathway between coronary artery bypass grafting (CABG) surgery type (off-pump vs on-pump), mediating variable acute kidney injury (AKI) and dependent variable renal function 1 year after surgery. The dashed arrows indicate that the causal relationship is unknown but will be investigated.

there is a differential impact of the intervention in those with and without CKD. We will interpret a $p$ value $\leq 0.05$ as statistically significant. Despite the size of CORONARY, there will only be adequate statistical power for very large subgroup effects.

\section{Mediation analysis: impact of AKI on longer term kidney function}

A mediating variable is one that explains all or part of the association between a predictor and a response. ${ }^{19}$ In CORONARY, mediation will occur if on-pump versus offpump CABG surgery influences the development of AKI, which influences 1-year kidney function. ${ }^{20}$ Regressionbased tests for mediation have been well developed and are widely used. ${ }^{21}{ }^{22}$ Figure 1 presents our analytic framework. We will test for a significant mediation effect (methods detailed in online appendix). We will report the proportion of the total effect of surgery type (offpump vs on-pump CABG) on per cent change in 1-year eGFR that is mediated by peri-operative per cent change in serum creatinine. As well, we will consider mediation with AKI defined as a categorical variable (methods detailed in online appendix).

\section{CONCLUSIONS}

The sample in CORONARY almost exceeds the combined number of patients enrolled across 70 randomised controlled trials, which tested strategies to prevent or treat AKI in cardiac surgery. ${ }^{23}$ It will be the largest AKI prevention trial conducted to date. International recruitment across 19 countries will provide generalisable estimates of the treatment effect. In this report, we have judiciously pre-specified the main questions and analytic protocol that will be used to test relevant kidney hypotheses in CORONARY. We have done so to minimise the chance of spurious post-hoc assertions of effect, so that the kidney results from this large international trial are robust and believable.

\footnotetext{
Author affiliations

${ }^{1}$ Division of Nephrology, Department of Medicine, Western University, London, Canada

${ }^{2}$ Department of Epidemiology and Biostatistics, Western University, London, Canada

${ }^{3}$ Department of Clinical Epidemiology and Biostatistics, McMaster University, Hamilton, Canada

${ }^{4}$ Population Health Research Institute, Hamilton, Canada
}

${ }^{5}$ Department of Statistics and Actuarial Science, University of Waterloo, Waterloo, Ontario, Canada

${ }^{6}$ Section of Nephrology, Yale University School of Medicine, New Haven, USA

${ }^{7}$ Division of Cardiac Surgery, McMaster University, Hamilton, Canada

${ }^{8}$ Department of Cardiac Surgery, Université de Montreal, Montreal, Canada

${ }^{9}$ Division of Cardiac Surgery, Western University, London, Canada

Contributors All the authors made substantial contributions to the conception and design, acquisition of data, and analysis and interpretation of data; drafting the article or revising it critically for important intellectual content and final approval of the version to be published.

Funding CORONARY and the CORONARY kidney substudy are funded by the Canadian Institutes of Health Research. The authors are solely responsible for the design and conduct of this trial, all analyses, the drafting and editing of the report, its final contents and the decision to submit for publication.

Competing interests None.

Ethics approval Ethics approval was provided by all participating trial centres.

Provenance and peer review Not commissioned; internally peer reviewed.

Data sharing statement No additional data are available.

\section{REFERENCES}

1. Hillis LD, Smith PK, Anderson JL, et al. 2011 ACCF/AHA Guideline for coronary artery bypass graft surgery: executive summary: a report of the American College of Cardiology Foundation/American heart association Task Force on Practice Guidelines. Circulation 2011;124:2610-42.

2. Fergusson DA, Hebert PC, Mazer CD, et al. A comparison of aprotinin and lysine analogues in high-risk cardiac surgery. $N$ Engl $J$ Med 2008;358:2319-31.

3. Chertow GM, Burdick E, Honour M, et al. Acute kidney injury, mortality, length of stay, and costs in hospitalized patients. J Am Soc Nephrol 2005;16:3365-70.

4. Mariscalco G, Lorusso R, Dominici $\mathrm{C}$, et al. Acute kidney injury: a relevant complication after cardiac surgery. Ann Thorac Surg 2011;92:1539-47.

5. Seabra VF, Alobaidi S, Balk EM, et al. Off-pump coronary artery bypass surgery and acute kidney injury: a meta-analysis of randomized controlled trials. Clin J Am Soc Nephrol 2010;5:1734-44.

6. Bucaloiu ID, Kirchner HL, Norfolk ER, et al. Increased risk of death and de novo chronic kidney disease following reversible acute kidney injury. Kidney Int 2012;81:477-85.

7. Coca SG, Singanamala S, Parikh CR. Chronic kidney disease after acute kidney injury: a systematic review and meta-analysis. Kidney Int 2012;81:442-8.

8. Gansevoort RT, Matsushita K, van der VM, et al. Lower estimated GFR and higher albuminuria are associated with adverse kidney outcomes. A collaborative meta-analysis of general and high-risk population cohorts. Kidney Int 2011;80:93-104.

9. Lamy A, Devereaux PJ, Prabhakaran D, et al. Rationale and design of the coronary artery bypass grafting surgery off or on pump Revascularization study: a large international randomized trial in cardiac surgery. Am Heart $J$ 2012;163:1-6.

10. Levey AS, Stevens LA, Schmid CH, et al. A new equation to estimate glomerular filtration rate. Ann Intern Med 2009;150:604-12.

11. Venkataraman R, Kellum JA. Defining acute renal failure: the RIFLE criteria. J Intensive Care Med 2007;22:187-93.

12. Mehta RL, Kellum JA, Shah SV, et al. Acute Kidney Injury Network: report of an initiative to improve outcomes in acute kidney injury. Crit Care 2007:11:R31.

13. National Kidney Foundation. K/DOQI clinical practice guidelines for chronic kidney disease: evaluation, classification, and stratification. Am J Kidney Dis 2002:39(2 Suppl 1):S1-266.

14. White IR, Thompson SG. Adjusting for partially missing baseline measurements in randomized trials. Stat Med 2005;24:993-1007.

15. Hauck WW, Anderson S, Marcus SM. Should we adjust for covariates in nonlinear regression analyses of randomized trials? Control Clin Trials 1998:19:249-56.

16. Gail MH, Wieand S, Piantadosi S. Biased estimates of treatment effects in randomized experiments with nonlinear regressions and omitted covariates. Biometrika 1984;71:431-44.

17. Holm S. A simple sequentially rejective multiple test procedure. $J$ Statist 1979;6:65-70.

18. Lassnigg A, Schmid ER, Hiesmayr M, et al. Impact of minimal increases in serum creatinine on outcome in patients after 
cardiothoracic surgery: do we have to revise current definitions of acute renal failure? Crit Care Med 2008;36:1129-37.

19. Baron RM, Kenny DA. The moderator-mediator variable distinction in social psychological research: conceptual, strategic, and statistical considerations. J Pers Soc Psychol 1986;51:1173-82.

20. Mackinnon DP, Warsi G, Dwyer JH. A Simulation study of mediated effect Measures. Multivariate Behav Res 1995;30:41.
21. Mackinnon DP, Lockwood CM, Hoffman JM, et al. A comparison of methods to test mediation and other intervening variable effects. Psychol Methods 2002;7:83-104.

22. Fritz MS, Mackinnon DP. Required sample size to detect the mediated effect. Psychol Sci 2007;18:233-9.

23. Park M, Coca SG, Nigwekar SU, et al. Prevention and treatment of acute kidney injury in patients undergoing cardiac surgery: a systematic review. Am J Nephrol 2010;31:408-18. 